\title{
Structure-Control Design of a Parallel Robot Based on Multi-Objective Self-Adaptive Differential Evolution Algorithm
}

\author{
Meng Qing Mei ${ }^{1, a}$, Sheng Hui Ping ${ }^{1, b}$ Zhong Ruo Bin ${ }^{1, c}$ and Pan Shi Yue \\ ${ }^{1}$ School of Mechanism Engineering, Changzhou, University \\ Changzhou, 213016, China \\ a mqmhit@sina.com, bshp@126.com, chasee500@163.com, ${ }^{\mathrm{d}} \mathrm{PSY} @ 126 . c o m$
}

\begin{abstract}
Keywords: Multi-objective optimization; differential evolution; dynamic optimization; parallel manipulator
\end{abstract}

\begin{abstract}
This paper presents a method based on a multi-objective self-adaptive differential evolution (MOSaDE) algorithm to improve the parametric reconfiguration feature in the optimal design of a parallel robot. We propose a MOSaDE algorithm, in which both trial vector generation strategies and their associated control parameter values are gradually self-adapted by learning from their previous experiences in generating promising solutions. Consequently, a more suitable generation strategy along with its parameter settings can be determined adaptively to match different phases of the search process. Furthermore, a constraint-handling mechanism is added to bias the search to the feasible region of the search space. The obtained solution will be a set of optimal geometric parameters and optimal PID control gains. The results obtained in a set of experiments performed mechatronic system show the effectiveness of the proposed approach.
\end{abstract}

\section{Introduction}

Parallel manipulators are closed-loop mechanical chains, which generally have good performance. Compared with serial robots, parallel mechanisms offer greater structural rigidity, lower moving mass, better accuracy, larger dynamic charge capacity, simpler modular mechanical construction. However, most existing parallel robots have limited and complicated workspace volume with singularities and highly non-isotropic input-output relations [1]. This is a very challenging task for a general parallel manipulator. In order to fulfill these requirements, a multidisciplinary analysis approach must be used in the design process of parallel manipulators systems [2]. The parametric optimal design of the mechatronic system needs to be stated as a constrained multi-objective dynamic optimization problem (MDOP). However, these methods present different shortcomings, e.g. they can be attracted by local minima in the neighborhood of the starting point. They are sensitive to the initial conditions and they involve the computation of the gradient and the Hessian of the objective function and constraints. In order to save these problem, non-traditional optimization techniques based on stochastic methods such as evolutionary algorithm (EA), genetic algorithms (GA) or particle swarm optimization (PSO) evolutionary algorithm can be used in order to overcome the above drawbacks. Recently, another search heuristic called differential evolution (DE) has shown performance superior to that of PSO and EA in the widely used benchmark problems. The main contributions of the present work is to state mechatronic design as a nonlinear dynamic optimization problem [3-4].

The purpose of this paper is to propose a constrained multi-objective dynamic optimization methodology to design the optimal parametric of the parallel mechanisms system. This methodology uses multi-objective self-adaptive differential evolution (MOSaDE) algorithm to obtain the set of optimal mechanical and controller parameters. The following section describes self-adaptive differential evolution approach to improve the reconfigurability feature in non-iterative concurrent design. The mechatronic system design and multi-objective dynamic optimization obtained are shown in Section 3. The results and discussion of the dynamic optimization problem are carried out in Section 4. Finally, the conclusions of this approach are presented. 


\section{Multi-objective Optimization Self-Adaptive Differential Evolution Algorithm.}

Differential evolution (DE) is an efficient and powerful population-based stochastic search technique for solving optimization problems over continuous space. In R. Storn [5] et al, They identified that DE could not search for some global optimum solution simultaneous. To improve the reliability of $\mathrm{DE}$ algorithm in dealing with multimodal optimization problem, we proposed the multi-objective self-adaptive differential evolution (MOSaDE) algorithm.

\section{Multi-objective Optimization.}

In real world applications, most optimization problems have complex constraints. A constrained optimization problem is usually written as a nonlinear programming. The Multi-objective optimization problem (MOP) can be formally defined as the problem of finding all $X\left(x_{1} \cdots x_{m}\right)$, which satisfied the $m$ inequality constraints: $g_{i}(X) \geq 0, t=1, \cdots, m$

$p$ equality constraints: $h_{f}(X)=0, f=\mathbb{1}_{v}, \cdots, p$

And optimize the vector function: $\operatorname{Min}\left(f_{1}(X), f_{2}(X) \cdots f_{k}(X)\right)$

In other words, the aim is to determine those decision vectors $X$ in the decision space $D$ which satisfy all the constraints and optimize the objective function vector.

\section{Trial Vector Generation Strategy Adaptation.}

In our proposed MOSaDE algorithm, the strategies are "rand/1/bin" and "best/2/bin", the $X_{\text {zerk }}$ in "best/2/bin" is randomly selected from external archive [5]. Since two candidate strategies are adopted, both strategies have equal probability to be applied to each individual in the initial population:

\section{$p_{1, \epsilon}=p_{2, \varepsilon}=0.5$}

Where $p_{1 .}$ is the probability of applying strategy "rand/l/bin" to each individual in the current population. $p_{2 c}$ is the probability of applying another strategy. After evaluation of all newly generated trial vectors, the number of trial vectors successfully entering the next generation while generated by the strategy "rand/1/bin" and the strategy "current to best/2/bin" are recorded as $n s_{1, \varepsilon}$, and $n s_{2, G}$, respectively, and the numbers of trial vectors discarded while generated by the strategy "rand/l/bin" and the strategy "current to best/2/bin" are recorded as $n f_{1, q}$ and $n f_{2, \varepsilon}$. Those two numbers are accumulated within a specified number of generations.

Then, the probability of $p_{1, g}$ is updated as:

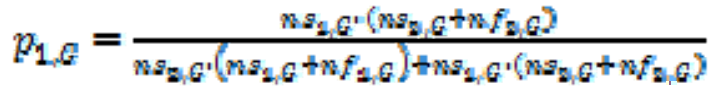

$$
\begin{aligned}
& p_{2, \epsilon}=1-p_{1, \epsilon}
\end{aligned}
$$

This adaptation procedure can gradually evolve the most suitable learning strategy at different learning stages for the problem under consideration. For each target vector $X_{t}^{\epsilon}$ at generation $G$, An associated mutated vector $V_{t}^{G}$ can usually be generated by using one of the adopted strategies:

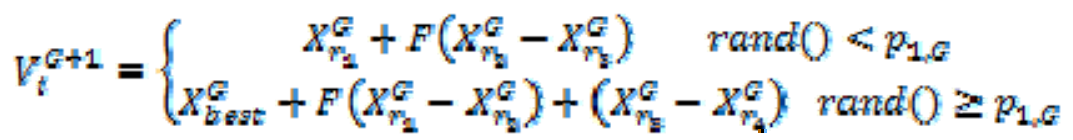

\section{Parameter Adaptation.}

In the conventional DE, the choice of numerical values for the three control parameters $F, C R$ and $N P$ highly depends on the problem under consideration. In our MOSaDE algorithm, we leave $N P$ as a user-specified parameter. Between the two parameters $C R$ and $F, C R$ is much more sensitive to the problem's property and complexity such as the multi-modality, while $F$ is more related to the convergence speed [6-8]. In order to adapt multi-target priority of demand, the number of non-dominated solutions were been dominated in the initial populations to maintain diversity of the population. Nevertheless, in the later stage of evolution, all individuals in the population are non-dominance relationship. In order to get better distribution, the diffused velocity of the individual to the sparse region was been increased.

The main steps of MOSaDE are described below: 
Step 1: Set generation counter $G=0$, the maximum generation $G e n=100$ and randomly initialize a

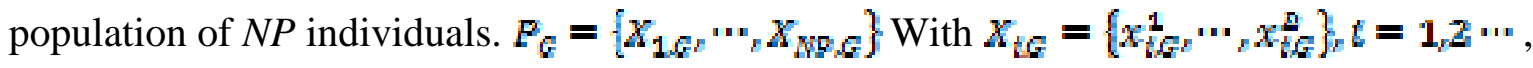

strategy probability $p_{1, e}=p_{2, e}=0.5$;

Step 2: Mutation operation. Generate a mutated vector $V_{t}^{G+1}$ for each target vector $X_{t}^{G+1}$ according equation (2);

Step 4: Selection operation. Based on the greedy search to generate $X_{l 6+1}$;

Step5: $G=G+1$, while stopping criterion $(G \propto G e n)$, return Step 2, otherwise output non-dominated solution set $Q$.

\section{Description of Manipulators System}

The manipulator comprises the closed-chain kinematic system depicted in Fig.1[9]. The manipulator consists of six revolute joints and 3 sliding pairs connected by 6 rigid links to form three linkages, two of which are actuated and connected to the base of robot. The manipulator is the parallel spraying robot with 2 DoFs, which can be used for positioning of desired point or for tracking of a desired trajectory on the XYZ plane.

\section{Parallel Manipulator Model.}

The kinematic equation maps the configuration space $Q$ into the task space $P\left(f: R^{m} \rightarrow R^{m}\right)$, where $n$ and $m$ represent the dimensions of the two spaces, respectively. In Fig. 1, given the vector of joint angles $₫=\left[Q_{2} s_{t}\right]^{T}$, where $\left(s_{i} t=1,2,3\right)$ represents the active joints and $\emptyset=\left[X_{W^{n}} Y_{M^{n}}, Z_{M}\right]$ is the manipulator displacement vector, the kinematic equations are given by

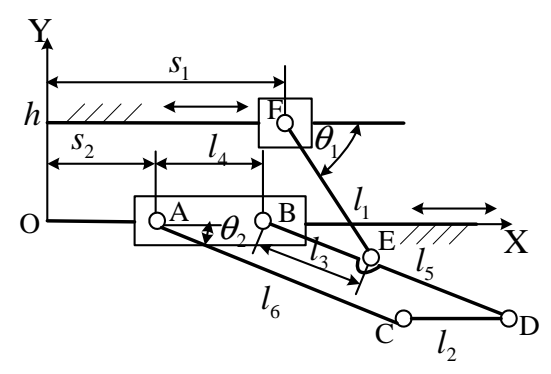

(a)Schematic manipulator
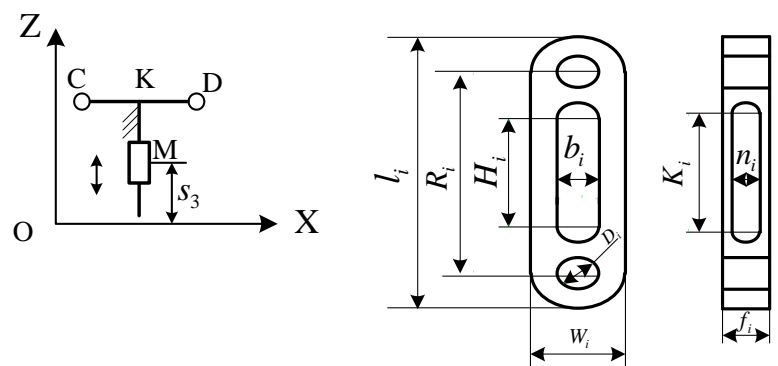

(b) Schematic representation of ith link

Fig.1 Schematic representation of manipulator

$$
\begin{aligned}
& X_{M}=s_{2}+l_{6} \cos \theta_{2}+\frac{l_{2}}{2} \\
& Y_{M}=l_{6} \sin \theta_{2} \\
& Z_{M}=s_{2}
\end{aligned}
$$

Where $l_{i}$ is the length of each link. It is important to note that in the kinematics of the manipulator might be considered as one of the task-space variables.

The Lagrangian formulation is employed to obtain the system dynamics.

$$
M(q) \ddot{q}+r(r, \dot{q}) \dot{q}+k q=u
$$

Where $M$ represent mass matrix , $C$ represent centrifugal and coriolis terms. $q=\left[q_{1}, q_{2}\right]^{2}$ and

$\grave{q}=\left[q_{1}, q_{2}\right]$ are independent generalized coordinate vectors of position and velocity of the actuated links.

Based on the idea of a mechatronic design, three PID controllers are chosen. According to the selection matrices in the two control loops guarantees that the position control is decoupled. Therefore, the control law for each loop can be designed independently.

\section{Optimization Problem.}

Let us consider Fig. 1of ith link is used because the geometric parameters of the links can modify in a wide range the dynamic parameters of the links and the kinematic parameters. On the other hand, 


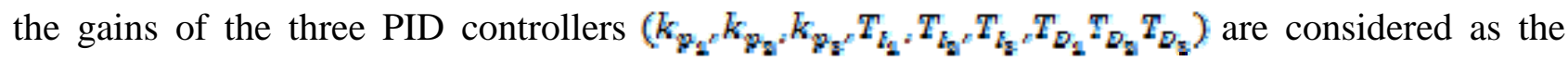
control design parameters to be optimized. So, the design parameter vector $p \in R^{\text {bq }}$ which involves structural and control design parameters results as in:

$p=\left[p_{g}, p_{g}\right]^{2}$

Where

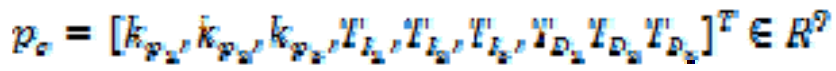

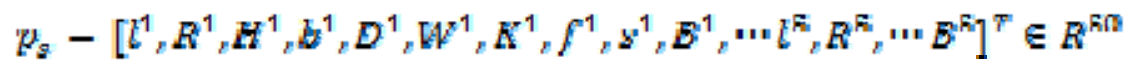

The MDOP consist of finding the optimal design variable vector $p$ which involves the optimal geometries of the parallel robot's links and the optimal PID controller gains. Since the control objective (to follow a desired point or trajectory) would not be able to be carried out. So, The MDOP can be formulated as follows:

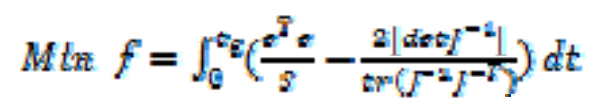

Where $e$ is the vector of angular position errors, $t_{s}$ is the finite time, $s$ is the square of the maximum angular position error found in the trajectory tracking for the time interval $\left[0, t_{z}\right], J$ is the Jacobian matrix of the parallel robot, $\operatorname{det}(\bullet)$ and $\operatorname{tr}(\bullet)$ are the determinant and the trace of the matrix $(\bullet)$.

\section{Experimental and Results}

In order to assess the performance of the proposed approach, which aims to favor reconfigurability in concurrent optimal mechatronic design by the introduction of MOSaDE method, the problems are solved by the adapted MOSaDE algorithm.

Based on the fact that the evaluation of one single solution takes several minutes, five independent runs were performed for each experiment; all independent runs were performed on the same computer platform. The fixed parameter values were the following for the parallel robot design: $N P=200, G E N=100$. The parameters for the parallel robot system were equal for $F$ and $C R$.

The different shapes of the parallel robot links with optimal design parameters obtained at each run. The optimal 1th link shape has a symmetrical appearance, as shown in Fig.3(a). The Fig.3(b) is parallel spraying robot.

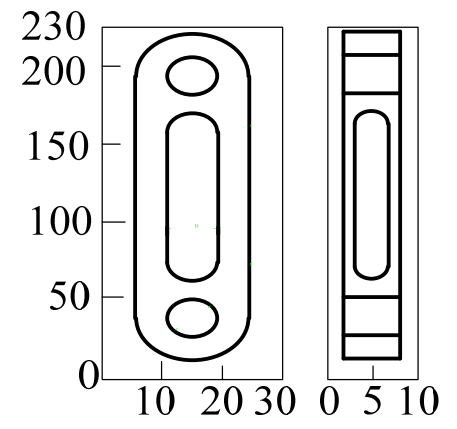

(a)Schematic of optimal structures of 1th links

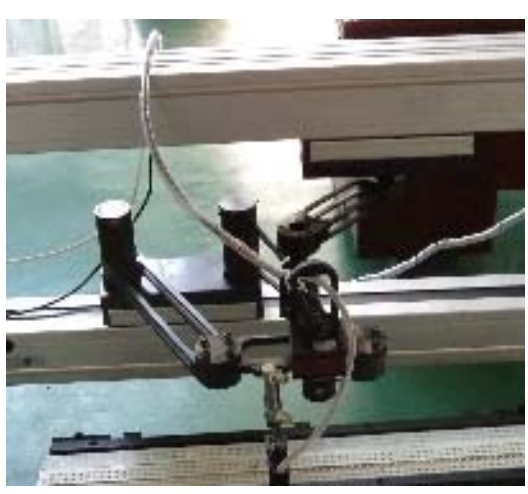

(b) parallel spraying robot

Fig.3 Behavior of the control signal

\section{Conclusions and Future Work}

In this article, a structure-control design for a parallel robot with trajectory tracking and singularity avoidance is stated. This is formulated as a nonlinear dynamic optimization problem, where the system dynamics (nonlinear differential equations) are considered as an equality constraint. This problem is solved by a constraint-handling self-adaptive differential evolution algorithm. The 
MOSaDE algorithm finds all design variables that improve a dynamic performance function value. The algorithm spends a reasonable time to converge to optimum solutions. In spite of having a large number of design variables and a highly constrained dynamic optimization problem, the algorithm works efficiently in searching for the optimum design variables.

The future paths of research consider the definition of an improved set of criteria which considers feasibility in the reconfigurability promotion mechanism.

\section{References}

[1] Pil.A. and Asada, H: Integrated Structure/Control Design of Mechatronics Systems using a Recursive Experimental Optimization Method. IEEE/ASME Transactions on Mechatronics. Vol. 1 (1996), p.191-203.

[2] Affi, Z, El-Kribi, B, and Romdhane,L: Advanced Mechatronic Design using a Multi-Objective Genetic Algorithm Optimization of a Motor-Driven Four-Bar System. Mechatronics, Vol.17 (2007), p. 489-500.

[3] Cruz-Villar, C.A, Alvarez-Gallegos and Villarreal-Cervantes: Concurrent Redesign of an Underactuated Robot Manipulator. Mechatronics, Vol. 19 (2009),p.178-183

[4] T. Matsumaru, Design and Control of the Modular Robot System: TOMMS, IEEE Int Conf on Robotics and Automation, Nagoya, Japan, 1995, pp. 2125-2131.

[5] R. Storn and K. V. Price: Differential Evolution-A Simple and Efficient Heuristic for Global Optimization over Continuous Spaces. J. Global Optim.Vol. 11(1997), p. 341-359.

[6] R. Storn and K. V. Price: Differential Evolution-A Simple and Efficient Heuristic for Global Optimization over Continuous Spaces. J. Global Optim.Vol. 11(1997), p. 341-359.

[7] Z. M. Bi and W. J. Zhang: Concurrent Optimal Design of Modular Robotic Configuration. Journal of Robotic Systems.Vol.18 (2001), p. 77-87.

[8] Saravanan, R and Ramabalan, S: Evolutionary Minimum Cost Trajectory Planning for Industrial Robots. Journal of Intelligent and Robotic System. Vol. 52(2008),p.45-77.

[9] Shen Huiping, Zhao Haibin. Deng Jiaming. Meng Qingmei and Zhu Wei : Type Design Method and the Application for Hybrid Robot Based on Freedom Distribution and Position and Orientation Characteristic Set. Journal of Mechanical Engingeering,Vol.47(2011),p.56-64 